\title{
Monocyte-mediated Serum-independent Damage to Hyphal and Pseudohyphal Forms of Candida albicans In Vitro
}

\author{
Richard D. Diamond and Christian C. HaudensChild, Evans Memorial \\ Department of Clinical Research and Department of Medicine, University \\ Hospital, and Department of Pathology, Boston University Medical Center, \\ Boston, Massachusetts 02118
}

\begin{abstract}
A B S T R A C T Human peripheral blood monocytes attached to Candida albicans hyphae in the absence of serum and damaged the hyphae without completely ingesting them. Attachment and damage was not augmented by the addition of serum. Damage to hyphae was quantitated by a previously developed metabolic assay that measured leukocyte-induced reduction in uptake of $\left[{ }^{14} \mathrm{C}\right]$ cytosine by the hyphae. Use of cells from patients with hereditary disorders of leukocyte function, chronic granulomatous disease, and myeloperoxidase deficiency indicated that myeloperoxidaseindependent and nonoxidative mechanisms could sometimes damage hyphae where oxidative mechanisms were impaired. Damage to hyphae by normal monocytes was inhibited by concentrations of sodium azide and sodium cyanide that primarily affect myeloperoxidase activity, as well as by halide-free conditions, catalase, and putative antagonists of hypochlorous acid or singlet oxygen. Iodination of hyphae, a myeloperoxidase and hydrogen peroxide-dependent process of monocytes, was similarly inhibited by sodium azide, sodium cyanide, and catalase. Under anaerobic conditions, damage to hyphae was reduced by $64.0-68.4 \%$. In contrast, inhibitors of potential nonoxidative antifungal mechanisms, iron salts to saturate iron chelators, and polyanionic amino acid polymers to neutralize cationic proteins did not block damage to hyphae by monocytes. Preparations rich in lysosomal granules from fractionated normal monocytes also did not damage hyphae. Overall, it appeared that oxidative mechanisms were most important for damage to hyphae by normal monocytes.
\end{abstract}

Dr. Diamond is a recipient of Research Career Development Award 00055 from National Institute of Allergy and Infectious Diseases, National Institutes of Health.

Received for publication 9 April 1980 and in revised form 12 August 1980.
Electron microscopy confirmed that Candida hyphae were damaged and probably killed by monocytes, but monocytes appeared to sustain significant damage in the process. In the absence of serum, monocyte cell membranes became closely approximated to Candida cell walls. It appeared that some Candida could escape this partial engulfment, as they were seen floating free with vesicular trilaminar membrane remnants covering hyphal surfaces. In general, monocytes appeared to be damaged by interactions with Candida hyphae more than neutrophils had been in previous studies.

\section{INTRODUCTION}

Invasive lesions caused by Candida albicans characteristically contain hyphal and pseudohyphal forms that are too large to be ingested by phagocytic cells. However, our previous studies established that these large forms of Candida could be damaged and probably killed by human neutrophils in the absence of complete ingestion $(1,2)$. Damage to hyphae was largely attributable to products of oxidative metabolism of neutrophils (2). Like neutrophils, monocytes and macrophages derived from them have been demonstrated to ingest and kill yeastlike forms of Candida (3-7). Mononuclear phagocytes may be important components of host defense mechanisms against invasive candidiasis by contributing to prevention of dissemination or by limiting the extent of mucocutaneous lesions. Defective function of mononuclear phagocytes may be involved in patients with the chronic mucocutaneous form of candidiasis (8). Several important host defense functions have been attributed to mononuclear phagocytes, including direct phagocytic microbicidal activity, antigen processing in the afferent limb of the immune response, interactions with lymphoid cells in cellular immune responses, and lysis of tumor cells 
by nonphagocytic killing mechanisms active at the cell surface (5). Though monocytes share many features with neutrophils, many differences between these cell types have become apparent. Unstimulated monocytes appear to have efficient intracellular microbicidal activity but have been found to be less actively phagocytic than neutrophils $(9,10)$. Though oxidative metabolism is largely responsible for microbicidal activity in both neutrophils and monocytes, production of oxidative products is not necessarily identical in both cell types $(9,11,12)$, and nonoxidative bactericidal activity of monocytes may be important in some situations $(3,13)$. From this, it might be inferred that monocytes could play an important role distinct from neutrophils in host defenses against candidiasis. Furthermore, the capacity for intracellular killing of ingested yeasts may not reflect the ability to damage hyphae at the monocyte surface by nonphagocytic mechanisms.

Therefore, in these studies, we determined that monocytes could damage and probably kill hyphae and pseudohyphae of $C$. albicans. Hyphal damage by normal monocytes appeared to be primarily due to products of oxidative metabolism, especially the myeloperoxidase- $\mathrm{H}_{2} \mathrm{O}_{2}$-halide system, but myeloperoxidase-independent mechanisms were active as well when myeloperoxidase-deficient monocytes were tested.

\section{METHODS}

Hyphae and pseudohyphae. An isolate of C. albicans originally obtained from a patient with systemic candidiasis was germinated in Eagle's minimal essential medium supplemented with nonessential amino acids (Gibco Laboratories, Grand Island Biological Co., Grand Island, N. Y.). Incubation at $37^{\circ} \mathrm{C}$ was continued until $\geq 95 \%$ of cells had formed germ tubes $\geq 30 \mu \mathrm{M}$ in length $(1,2)$.

Monocytes. Mixed mononuclear cells were obtained from human peripheral blood by sedimentation on a Ficoll(Pharmacia Fine Chemicals, Inc., Piscataway, N. J.) Hypaque (sodium and meglumine diatrizoates, Winthrop Laboratories, N. Y.) gradient, followed by dextran sedimentation to obtain neutrophils for some studies (14). Preparations of mononuclear cells were adjusted according to the relative percentages of monocytes, which varied from $18-42 \%$ as judged by neutral red ingestion and fluorescent staining with acridine oragne. Mononuclear cell preparations used in these studies contained $1.0 \%$ neutrophils. For selected experiments, more purified preparations of monocytes were obtained by counterflow centrifugation (Beckman J21B with elutriator rotor; Beckman Instruments, Inc., Fullerton, Calif.) (15). These preparations contained 91-94\% monocytes by nonspecific esterase staining (16). Monocytes were $98.5-100 \%$ viable as judged by trypan blue exclusion. Monocytes were depleted from preparations by passage through nylon fiber columns (17) or by depletion of adherent cells by passage through three sets of plastic tissue culture dishes. Children with typical chronic granulomatous disease (three sex-linked, one autosomal recessive) and adults with hereditary myeloperoxidase deficiency were identified and blood was kindly supplied by Dr. Robert A. Clark (University Hospital, Boston, Mass.), Dr. Harvey Cohen (Children's Hospital, Boston, Mass.), and
Dr. Richard K. Root (Yale-New Haven Hospital, New Haven, Conn.).

Sera. For selected studies, autologous sera were obtained from normal volunteer subjects at the same time as monocytes. Serum obtained from a patient with disseminated candidiasis had anti-Candida antibodies detectable by immunodiffusion and a Candida whole cell agglutination titer of 1:128. Rabbit antiserum to Candida had a 1:512 agglutination titer.

Separation of subcellular fractions of monocytes. Mononuclear cells were lyzed and subcellular fractions were isolated according to the method of Kimball et al. (18). Pelleted Hypaque-Ficoll separated mononuclear cells were washed in $5 \mathrm{ml} 0.34 \mathrm{M}$ cold sucrose, then centrifuged at $4^{\circ} \mathrm{C}$ for $5 \mathrm{~min}$ at $1,000 \mathrm{~g}$. Cells were resuspended in $2 \mathrm{ml}$ of 0.34 $M$ cold sucrose containing 5,000 U heparin. Suspensions were repeatedly suctioned through Pasteur pipettes until $\geq 95 \%$ of cells were broken, as judged by phase contrast microscopy. An additional $10 \mathrm{ml}$ of cold $0.34 \mathrm{M}$ sucrose were added to lysates and gently but evenly mixed. $2 \mathrm{ml}$ were removed and mixed with $4 \mathrm{ml}$ of $0.34 \mathrm{M}$ sucrose (designated as the lysate fraction). The remaining lysate was centrifuged at $800 \mathrm{~g}$ for 10 min., the supernate $\left(S_{1}\right)$ saved, and the pellet resuspended in $5 \mathrm{ml} 0.34 \mathrm{M}$ sucrose and centrifuged at $400 \mathrm{~g}$ for $5 \mathrm{~min}$. This supernate $\left(S_{2}\right)$ was saved, and the pellet mixed with $6 \mathrm{ml} 0.34$ $M$ sucrose (designated as the nuclear fraction). Supernates $\left(S_{1}\right.$ and $\mathrm{S}_{2}$ ) were combined and centrifuged at $27,000 \mathrm{~g}$ for $20 \mathrm{~min}$, pellets were resuspended in $6 \mathrm{ml}$ of $0.34 \mathrm{M}$ sucrose (designated as the granular fraction), and remaining supernates that contained no free granules or other debris were designated as the post-granular supernate. For some experiments, heparin in fractions was neutralized by addition of protamine sulfate (Sigma Chemical Co., St. Louis, Mo.). Fractions were used fresh or stored at $-20^{\circ} \mathrm{C}$ until assayed for their ability to damage Candida hyphae. For assays of damage to hyphae, some batches of fractions were sonicated or subjected to repeated freezing and thawing in dry ice and methanol in order to ensure lysis of granules and release of granule contents before use.

Assessment of hyphal damage by uptake of $\left[{ }^{14} \mathrm{C}\right]$ cytosine. Damage to hyphae was measured as in previous studies (1). Briefly, suspensions containing $5 \times 10^{5}$ Candida albicans hyphae and $5 \times 10^{6}$ monocytes in $0.5 \mathrm{ml}$ Hanks' balanced salt solution were placed in 15 -ml plastic centrifuge tubes (Corning Glass Works, Science Products Div., Corning, N. Y.). In some studies, monocytes were replaced by separated subcellular fractions of monocytes. Control tubes contained $5 \times 10^{5}$ hyphae without leukocytes or subcellular fractions. Triplicate tubes were rotated for $1 \mathrm{~h}$ at $37^{\circ} \mathrm{C}$. Control tubes then received $5 \times 10^{6}$ leukocytes or equivalent amounts of subcellular fractions present in experimental tubes. Selected assays were performed anaerobically in triplicate $15-\mathrm{ml}$ polycarbonate plastic tubes with tightly fitting stoppers and in triplicate anaerobic specimen collectors (Becton, Dickinson \& Co., Rutherford, N. J.). Tubes were flushed with highly purified nitrogen that was passed first through an alkaline pyrogallol, then distilled water. Hanks' balanced salt solution was kept under vacuum overnight, followed by bubbling through with purified nitrogen for $4 \mathrm{~h}$ before use. Sets of control tubes were included to monitor $\mathrm{pH}$, oxygen content and oxygen consumption in response to ingestion of opsonized zymosan (YSI model 53 oxygen monitor, Yellow Springs Instrument Co., Yellow Springs, Ohio). Compared with airsaturated media, these conditions reduced oxygen content to $<1 \%$, and there was no stimulation in oxygen consumption with ingestion of opsonized zymosan by leukocytes. Leukocytes in all tubes were then lyzed with $2.5 \%$ sodium deoxycholate. Remaining hyphae were washed twice in distilled water and once in yeast nitrogen base broth that had been 
supplemented to include $1 \%$ dextrose and $0.15 \%$ asparagine. Each tube then received $0.25 \mu \mathrm{Ci}$ of $\left[{ }^{14} \mathrm{C}\right]$ cytosine (Research Products International Corp., Elk Grove Village, Ill.) dissolved in supplemental yeast nitrogen base broth. After incubating at $30^{\circ} \mathrm{C}$ for $1 \mathrm{~h}$, hyphae were washed free of unbound $\left[{ }^{14} \mathrm{C}\right]$ cytosine using an automated multiple sample harvester (Otto Hillar Co., Madison, Wis.). Filters were dried and counted in a liquid scintillation counter. Percentage of reduced uptake of $\left.{ }^{[14} \mathrm{C}\right]$ cytosine by hyphae induced by monocytes or subcellular fractions was calculated from (mean counts per minute in control tubes) - (mean counts per minute in experimental tubes $) /($ mean counts per minute in control tubes) $\times 100$.

Iodination. Fixation of ${ }^{125}$ I to Candida by monocytes was measured according to the methods of Klebanoff and Clark (19) with the minor modifications outlined in previous studies (1). Triplicate incubation tubes each contained $1 \times 10^{7}$ monocytes and $5 \times 10^{6} \mathrm{Candida}$ hyphae and $0.5 \mu \mathrm{M}(0.2 \mu \mathrm{Ci}) \mathrm{Na}^{125} \mathrm{I}$ (New England Nuclear, Boston, Mass.) and were tumbled at $37^{\circ} \mathrm{C}$ for $1 \mathrm{~h}$.

Inhibitors. Catalase (bovine liver, $6.1 \mathrm{mg} / \mathrm{ml}, 50,000$ $\mathrm{U} / \mathrm{mg}$ ) was obtained from Worthington Biochemical Corp. (Freehold, N. J.) and dialyzed against water before use. Superoxide dismutase (bovine erythrocyte, lyophilized powder,

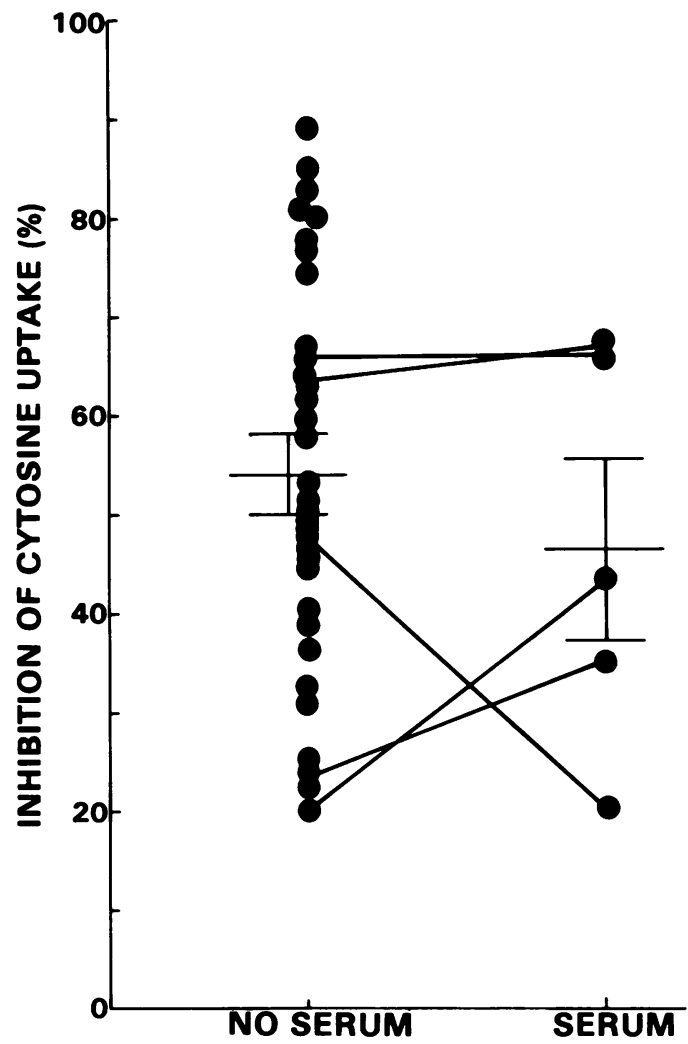

Figure 1 Damage to hyphae by monocytes from normal subjects in the presence and absence of $10 \%$ autologous serum. Hyphal damage was assessed by inhibition of $\left[{ }^{14} \mathrm{C}\right]$ cytosine uptake. Each dot denotes monocytes from a different subject. Results with monocytes tested simultaneously with and without serum are connected by straight lines. Mean \pm SEM are indicated by horizontal lines, and are connected by vertical lines.
12,300 U/mg) obtained from Miles Laboratories Inc., Research Products (Elkhart, Ind.) was dissolved in water at a concentration of $5 \mathrm{mg} / \mathrm{ml}$ and stored at $-20^{\circ} \mathrm{C}$ until used. For use in incubations, catalase and superoxide dismutase were first incubated with hyphae at $37^{\circ} \mathrm{C}$ for $1 \mathrm{~h}$ before addition of leukocytes, as these substances were noninhibitory without this preincubation. For some experiments, catalase was heated at $100^{\circ} \mathrm{C}$ for $15 \mathrm{~min}$., and superoxide dismutase was autoclaved at $121^{\circ} \mathrm{C}$ for $30 \mathrm{~min}$ before use. Sodium azide, sodium cyanide, histidine, tryptophan, dimethyl sulfoxide (DMSO), ${ }^{1}$ mannitol, sodium benzoate, ferric chloride, ferric and ferrous ammonium sulfate, and polymerized amino acids (polyaspartic acid, polyglutamic acid, polyarginine, and polylysine) were obtained from Sigma Chemical Co. Triethylenenediamine, also known as 1,4-diazobicyclo[2,2,2]octane (DABCO) was supplied by Eastman Organic Chemicals Division (Eastman Kodak Co., Rochester, N. Y.).

Electron microscopy. After $60 \mathrm{~min}$ of incubation with monocytes, hyphae and monocytes were fixed in suspension by addition of phosphate-buffered glutaraldehyde/formaldehyde (final concentrations 1 and $4 \%$, respectively). For preparation for transmission microscopy, the cells were spun at $450 \mathrm{~g}$ for $10 \mathrm{~min}$ and resuspended in the next solution for each of the following steps: $1 \%$ aqueous osmium tetroxide for $60 \mathrm{~min}$, $0.1 \%$ aqueous uranyl acetate for $30 \mathrm{~min}$, graded alcohols, propylene oxide and Epon 812 (Shell Chemical Co., Houston, Tex.). Toluidine blue stained sections, $1-\mu \mathrm{M}$ thick, were cut in both a plane perpendicular and a plane parallel to the axis of g-forces of centrifugation to examine the distribution of cells within the pellet. Ultrathin sections through the center of the pellet (including all cells from top to bottom) were cut with diamond knives, stained with lead citrate and uranyl acetate, and examined with a Philips EM 300 at $80 \mathrm{kV}$ (Philips Electronic Instruments, Inc., Mahwah, N. J.).

Statistical methods. Mean \pm SEM were compared using the two-tailed two sample $t$ test (20).

\section{RESULTS}

Metabolic assays for damage to hyphae by monocytes. C. albicans hyphae were damaged by human peripheral blood monocytes, as judged by monocyteinduced reduction in uptake of $\left[{ }^{14} \mathrm{C}\right]$ cytosine by the hyphae (Fig. 1). As was the case with neutrophils (1), monocyte-mediated reduction in $\left[{ }^{14} \mathrm{C}\right]$ cytosine uptake correlated with changes in uptake of $\left[{ }^{3} \mathrm{H}\right]$ glucose and mannose, as well as electron-microscopic evidence to damage to the fungi. Monocytes rather than lymphocytes mediated the damage to hyphae, as lymphocytes depleted of monocytes by passage through nylon wool columns or by adherence to plastic dishes did not damage Candida at all. Furthermore, hyphal damage by preparations depleted of more than $90 \%$ of lymphocytes by elutriator (15) was no greater than by equal numbers of Hypaque-Ficoll separated monocytes consisting of $60-80 \%$ lymphocytes $(45.0 \pm 5.9 \%$ damage by elutriator monocytes vs. $42.9 \pm 6.3 \%$ by Hypaque-Ficoll monocytes in three experiments). As in previous studies with neutrophils (1), damage to hyphae by monocytes was

${ }^{1}$ Abbreviations used in this paper: DABCO, 1,4-diazobicyclo[2,2,2]octane: DMSO, dimethyl sulfoxide. 
irreversible and was complete within $30-60 \mathrm{~min}$. Unlike previous observations using neutrophils (1), attachment to and damage of hyphae by monocytes was not augmented by the addition of autologous or homologous human sera (Fig. 1). Similarly, human and rabbit sera containing anti-Candida IgG antibodies affected neither attachment to hyphae as judged by phase contrast microscopy, nor damage to hyphae determined by cytosine uptake. Accordingly, all subsequent experiments were performed in the absence of serum.

Mechanisms of damage to hyphae by monocytes. Previous studies had shown that oxidative metabolism by neutrophils was critical in affecting damage to $C$. albicans hyphae (1). To study the importance of these processes in monocyte-mediated damage, cells were first used from patients with two well-characterized dysfunctions of phagocytic cells. In hereditary chronic granulomatous disease, particles are ingested normally by neutrophils and monocytes. However, the normal postphagocytic burst in oxygen consumption does not occur and potentially microbicidal products are not produced in normal amounts, including hydrogen peroxide, superoxide anion, and perhaps other oxidative intermediates as well (12). In hereditary myeloperoxidase deficiency, particle ingestion is also normal and hydrogen peroxide is produced, but myeloperoxidase is not present to react with peroxide and a halide to produce potent microbicidal substances (12). Previous studies (21) showed that neutrophils from two patients with myeloperoxidase deficiency and three (two sex-linked, one autosomal) of four patients with chronic granulomatous disease failed to damage Candida hyphae at all. Neutrophils from the fourth patient with chronic granulomatous disease (typical, sex-linked) damaged hyphae minimally, below values obtained with leukocytes from normal control subjects (Fig. 2). Damage to hyphae by monocytes from this same patient was in the upper part of the range for normal subjects. Shortly after testing, this subject received a bone marrow transplant, preventing performance of repeat studies. Monocytes from the other three subjects with chronic granulomatous disease did not cause detectable damage to hyphae. In contrast, monocytes from the two patients with myeloperoxidase deficiency caused significant damage to Candida hyphae, below mean values for normal subjects, but within the normal range (Fig. 2). These results are consistent with a central importance for oxidative mechanisms in damage to Candida by monocytes. However, nonmyeloperoxidase-dependent mechanisms appeared to have been active in myeloperoxidase-deficient monocytes, and nonoxidative mechanisms may have played a role in damage to hyphae by monocytes from one of the four patients with chronic granulomatous disease.

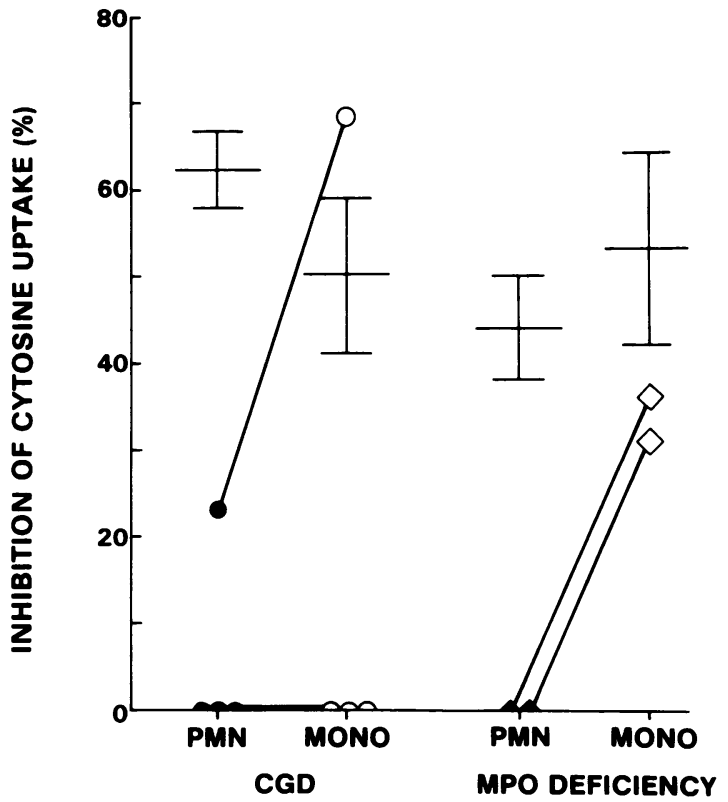

Figure 2 Damage to Candida hyphae by leukocytes from patients with chronic granulomatous disease, myeloperoxidase deficiency, and normal subjects. Hyphal damage was quantitated by leukocyte-induced inhibition of uptake of $\left[{ }^{14} \mathrm{C}\right]$ cytosine. Results are shown as individual points for each set of neutrophils (PMN; O) and monocytes (MONO; $O$ ) from each of the four patients with chronic granulomatous disease (CGD), and neutrophils $(\diamond)$ and monocytes $(\diamond)$ from each of the two patients with myeloperoxidase (MPO) deficiency. As shown, neutrophils and monocytes from three of the four CGD patients and neutrophils from both patients with MPO deficiency did not damage hyphae at all. These results for neutrophils obtained in our previous studies (21) are connected by solid lines to points obtained using the same patients' monocytes. Superimposed are horizontal lines connected by centered perpendicular lines, denoting mean \pm SEM for leukocytes from normal volunteer subjects. Normal leukocytes were tested simultaneously as controls with each set of CGD and MPO-deficient cells.

To assess the relative roles of these mechanisms in damage to hyphae by normal monocytes, inhibitors of monocyte function were tested for their ability to block the damage to hyphae which occurred in the absence of inhibitors. In the concentrations used in these studies, the inhibitors impaired neither viability (as judged by trypan blue exclusion) nor oxygen consumption by monocytes. Sodium azide and sodium cyanide inhibited damage to hyphae by monocytes (Table I). As shown by Klebanoff (22), the concentrations used primarily affect myeloperoxidase activity. Specificity of inhibition was confirmed using monocytes from one patient with myeloperoxidase deficiency, which damaged hyphae $(\geq 36.2 \%)$ equally well in the presence or absence of $0.1 \mathrm{mM}$ sodium azide. Omission of halide or addition of catalase inhibited monocyte-mediated 
TABLE I

Effects of Potential Inhibitors of Oxidative Microbicidal Mechanisms of Leukocytes on Damage to Candida albicans Hyphae by Monocytes

\begin{tabular}{lcc}
\hline \multicolumn{1}{c}{ Inhibitor (concentration added) } & Inhibition* & $\begin{array}{c}\text { Number of } \\
\text { experiments }\end{array}$ \\
\hline & $\%$ & \\
Sodium azide $(0.1 \mathrm{mM})$ & $47.1 \ddagger$ & 6 \\
Sodium cyanide (1.0 mM) & $49.8 \ddagger$ & 4 \\
Halide-free system (phosphate- & & \\
$\quad$ bicarbonate buffer) & 100.0 & 2 \\
Catalase (2,100 U) & $50.1 \pm 6.3$ & 6 \\
Superoxide dismutase (25 $\mu \mathrm{g})$ & 0.0 & 3 \\
DABCO (0.1 mM) & $19.0 \ddagger$ & 2 \\
Histidine (0.1 mM) & $20.5 \ddagger$ & 2 \\
Histidine (1.0 mM) & $39.8 \ddagger$ & 2 \\
Histidine (10.0 mM) & $91.6 \ddagger$ & 2 \\
Tryptophan (1 mM) & 2.4 & 2 \\
Tryptophan (10 mM) & $89.4 \ddagger$ & 2 \\
DMSO (14 mM) & 12.7 & 2 \\
DMSO (1.4 mM) & 10.0 & 2 \\
\end{tabular}

* Calculated from results of $\left[{ }^{14} \mathrm{C}\right]$ cytosine uptake in the presence and absence of inhibitors. Values represent means of at least two separate experiments, each performed in triplicate.

$\downarrow P<0.05$ by two sample $t$ tests.

damage to hyphae but superoxide dismutase did not inhibit damage to hyphae at all. Other products may also be derived from oxidative metabolism of neutrophils and monocytes. Three putative quenchers of singlet oxygen (also antagonists of hypochlorous acid), DABCO (23), histidine (24), and tryptophan (25), all inhibited damage to hyphae. DABCO was least effective but could not be used in higher concentrations, where it caused damage to hyphae by itself, in the absence of monocytes. DMSO (26), a hydroxyl radical scavenger and antioxidant, had only a minimal, insignificant effect on damage to hyphae by monocytes. Other less potent putative hydroxyl radical scavengers (sodium benzoate, mannitol) were also noninhibitory. Prolongation of incubations beyond one hour did not change sensitivity of the inhibitors tested.

Further support for the importance of myeloperoxidase-mediated damage to hyphae by monocytes was provided by quantitation of iodination (Table II). On contact with hyphae, myeloperoxidase is released from monocyte granules and is free to react with hydrogen peroxide produced by the monocytes. This results in the fixation of added iodide to fungal proteins, measurable as trichloroacetic acid precipitable ${ }^{125}$ I. Hyphae were iodinated by monocytes and this process was inhibited by azide, cyanide and catalase. Anaerobic experiments provided additional evidence for the im-
TABLE II

Effects of Inhibitors of Myeloperoxidase-dependent Microbicidal Mechanisms of Leukocytes on Iodination of Candida Hyphae by Monocytes

\begin{tabular}{cc}
\hline Inhibitor (concentration added) & Inhibition $^{*}$ \\
\hline$\%$
\end{tabular}

$\begin{array}{ll}\text { Sodium azide }(0.1 \mathrm{mM}) & 96.0 \pm 2.8 \ddagger \\ \text { Sodium cyanide }(1.0 \mathrm{mM}) & 94.8 \pm 1.7 \ddagger \\ \text { Catalase }(2,100 \mathrm{U}) & 61.6 \pm 8.9 \ddagger\end{array}$

* Calculated from results using monocytes from four different subjects, where mean $\left( \pm\right.$ SEM) fixation of ${ }^{125}$ I to hyphae was $21,769( \pm 2,624) \mathrm{cpm}$ above values for control tubes which contained hyphae but no monocytes.

$\ddagger P<0.05$ by two sample $t$ test.

portance of oxidative mechanisms in damage to hyphae by monocytes. In three experiments, each performed in two different types of anaerobic environments, damage to Candida hyphae was significantly reduced compared with simultaneous aerobic control incubations $(24.9 \pm 3.6 \%$ damage vs. $69.1 \pm 0.9 \%$ aerobically, and $10.6 \pm 7.8$ vs. $33.4 \pm 5.4 \%$ aerobically, reductions in damage of 64.0 and $68.4 \%$, respectively).

These results do not exclude the possibility that nonoxidative mechanisms may also be active in monocytemediated damage to fungi. Two potential antifungal mechanisms were studied using inhibitors: iron chelation and cationic proteins. Neither addition of excess iron salts ( $10 \mathrm{mM}$ ferric chloride, ferric ammonium sulfate, and ferrous ammonium sulfate) nor of polyanionic polymerized amino acids ( $10 \mu \mathrm{M}$ polyaspartic and polyglutamic acids) significantly inhibited damage to hyphae by monocytes. Other polyanions, such as heparin and $\alpha_{1}$-antitrypsin, were nonspecific inhibitors of oxidative metabolism and could not be used (2). However, it remained possible that monocyte granule constituents had antifungal effects by other mechanisms or that inhibitors could not reach sites of action due to the close proximity of monocyte and hyphal surfaces. Therefore, monocytes were fractionated and granule-rich preparations were obtained. Neither whole cell lysates nor granule-rich fractions damaged hyphae. Nonspecific inhibition of cationic proteins was not apparent, as there was $88.2 \pm 3.3 \%$ damage to hyphae by the nuclear pellet, rich in cationic histones which have antifungal activity (27).

Ultrastructural observations of monocyte-hypha interactions. Electron microscopy was used to examine the effects of contact between monocytes and Candida hyphae. Contamination of specimens with neutrophils was $1.0 \%$ or less. When samples were taken 1 min after adding hyphae to the monocytes, no contact 


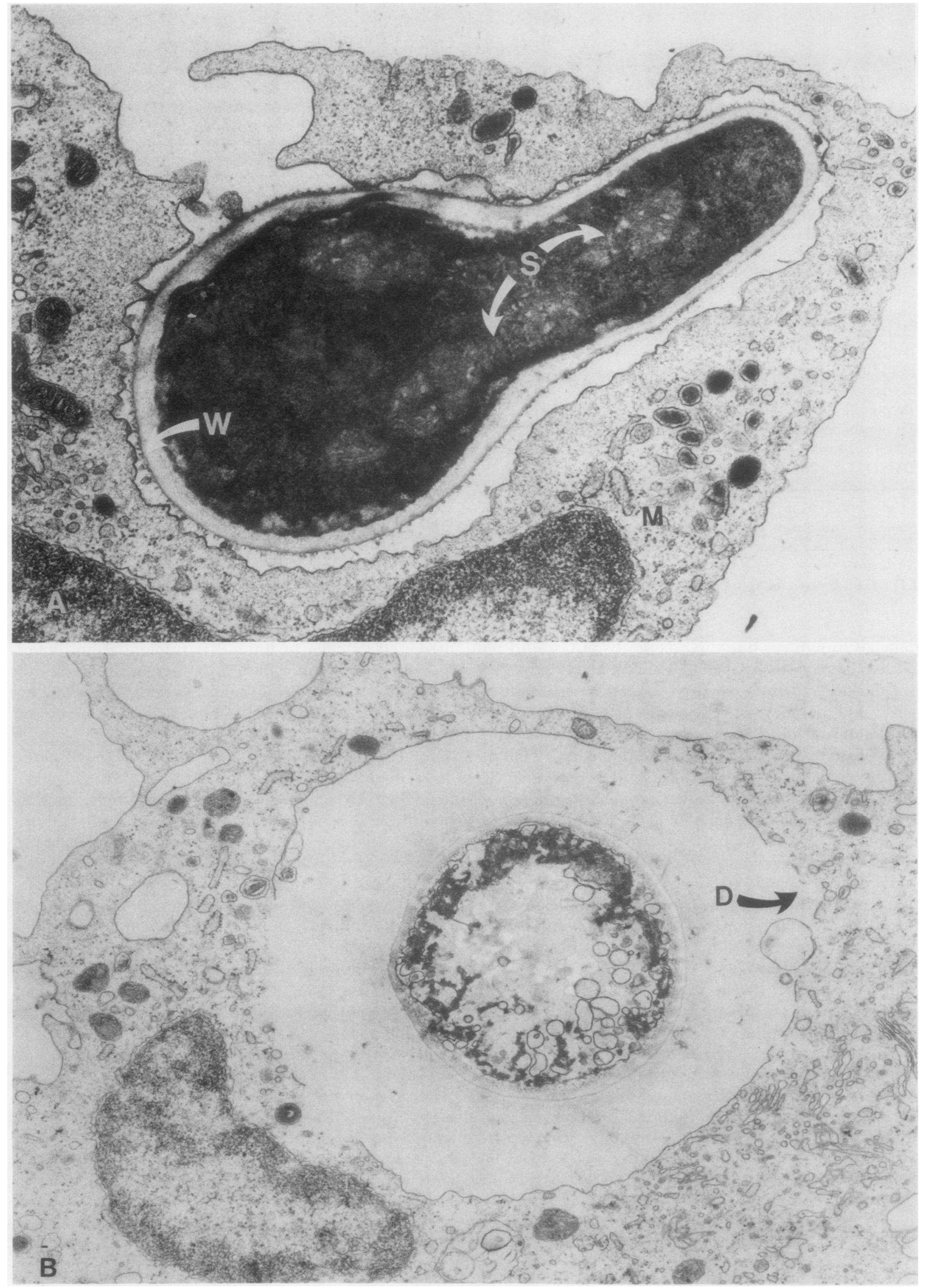



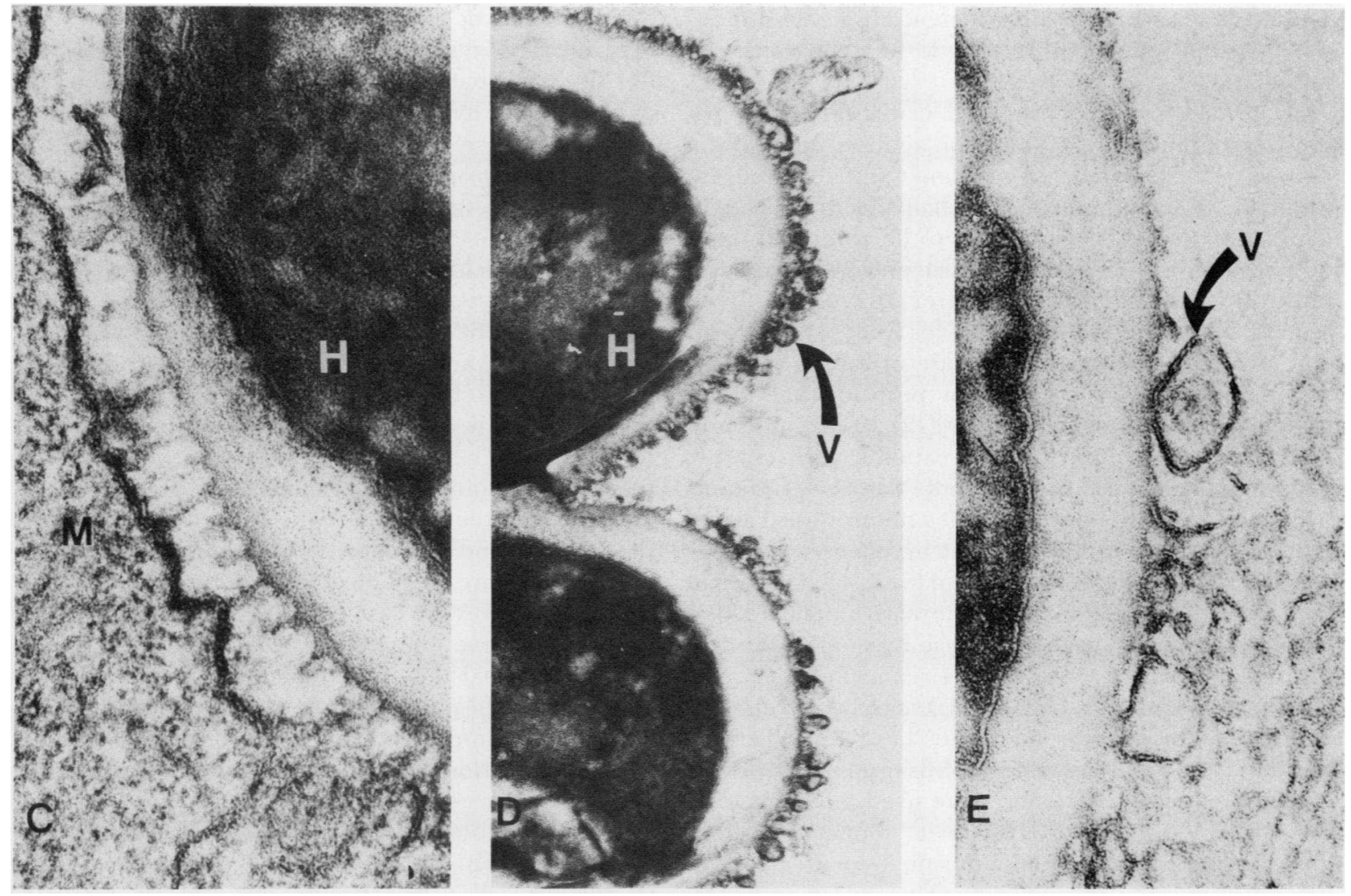

FIGURE 3 Electron micrographs showing interactions of hyphae with monocytes. (A) Monocyte (M) partially engulfing Candida hyphae with intact cell wall (W) and internal structures (S). $\times 35,112$. (B) Cross-section of ingested hypha with disrupted internal structures and thinning of cell wall, surrounded by a monocyte which is swollen and exhibits membrane disruption (D). $\times 21,033$. (C) Area of close contact between plasma membrane of monocyte (M) to Candida hyphal $(\mathrm{H})$ cell wall. $\times 151,335$. (D) Candida hypha $(\mathrm{H})$ surrounded by vesicular structures $(\mathrm{V})$. $\times 51,414$. (E) Magnified view of hyphal (H) surface showing adherent trilaminar vesicular membranes $(V) . \times 141,645$.

between hyphae and monocytes was seen and both monocytes and hyphal morphology appeared normal. After $1 \mathrm{~h}$ of incubation of monocytes with hyphae, the fungi were seen in various stages of partial engulfment by monocytes. Some of these partially engulfed fungi still appeared normal, with relatively intact internal structures (Fig. 3A). Other fungi had definite disruption of internal structures. At least $70 \%$ of monocytes that surrounded these damaged hyphae and some monocytes that surrounded intact hyphae were swollen and showed membrane disruption (Fig. 3B). Where no Candida were in contact, virtually none of the monocytes appeared swollen or damaged. The serum-independent contact between monocytes and hyphae involved close approximations between electron-dense portions of Candida cell walls with plasma membranes of monocytes (Fig. 3C). Some free-floating as well as engulfed Candida hyphae were covered with apparently surface-adherent vesicles and remnants (Fig. 3D) that had a trilaminar unit membrane structure (Fig. 3E). No such structures were observed on the surface of hyphae that had been incubated for only $1 \mathrm{~min}$ with monocytes. In contrast to monocytes, no damage to hyphae was attributable to contact with lymphocytes.

\section{DISCUSSION}

These studies have established that human peripheral blood monocytes could damage and probably kill the large tissue-invasive forms of C. albicans in the presence of complete ingestion. The mean amount and range of damage to hyphae by monocytes from normal subjects was almost identical to that observed in prior studies where normal neutrophils were used (1). With both cell types, attachment to hyphae occurred in the absence of serum. However, while IgG in serum aug- 
mented attachment of hyphae to neutrophils (1), no consistent serum effects on monocyte function were observed.

Experiments were performed using cells from patients with leukocyte dysfunction and leukocytes from normal subjects incubated with and without various inhibitors. In many respects, mechanisms of damage to hyphae by monocytes appeared to be similar to those observed in our previous experiments using neutrophils $(2,21)$, but significant differences were observed as well. Neutrophils and monocytes from patients with chronic granulomatous disease are unable to produce adequate amounts of hydrogen peroxide and other products of oxidative metabolism (12). Neutrophils and monocytes from three of four patients with chronic granulomatous disease did not damage Candida hyphae at all. The fourth patient's neutrophils caused minimal damage to hyphae, but his monocytes damaged hyphae in the high portion of the range for normal leukocytes. This suggests that alternative nonoxidative mechanisms for damage to hyphae by monocytes may become operative in some circumstances. Comparable nonoxidative bactericidal activity by chronic granulomatous disease monocytes has been observed by others $(13 ; 28)$. When neutrophils from patients with myeloperoxidase deficiency were used, no damage to hyphae was detected. However, monocytes from these patients caused significant hyphal damage, though below the mean level for normal monocytes. Myeloperoxidase-mediated microbicidal activity occurs through interactions with hydrogen peroxide and a halide (22) and is of central importance in intracellular killing of $C$. albicans yeasts $(3,29)$. In our previous studies using cell-free systems, the myeloperoxidase-peroxide-halide system damaged Candida hyphae efficiently (21). However, under some circumstances, such as use of other species of Candida yeasts, myeloperoxidase-independent fungicidal mechanisms may be operative $(3,30)$. Such mechanisms appeared to be operative in our studies of damage to C. albicans hyphae by myeloperoxidase-deficient monocytes.

Use of inhibitors of leukocyte function provided additional insights into the mechanisms of hyphal damage by normal monocytes. Azide and cyanide (used in concentrations that primarily affected myeloperoxidase [22]), as well as catalase and halide-free conditions, significantly inhibited damage to hyphae and iodination of hyphae by monocytes. Superoxide dismutase failed to inhibit hyphal damage, so superoxide anion may lead more to production of hydrogen peroxide rather than a direct antifungal effect. Both superoxide anion and hydrogen peroxide are known to be produced by monocytes and macrophages (12, $31-33)$. Other potential antimicrobial substances also may be produced by oxidative metabolism of stimu- lated phagocytic leukocytes, including hypochlorous acid, singlet oxygen and hydroxyl-free radical $(11,12$, $31,34-36$ ). Putative quenchers of singlet oxygen (also antagonists of hypochlorous acid), DABCO (23), histidine (24), and tryptophan (25), all caused significant inhibition of damage to Candida hyphae by monocytes. While singlet oxygen produced by monocytes may then contribute to damage of hyphae, the nonspecificity of these quenching agents $(34,35)$ may likely reflect damage by hypochlorous acid rather than by singlet oxygen (34-36). DMSO, a potent hydroxyl radical scavenger (26) and an antioxidant as well, did not significantly inhibit damage to hyphae by monocytes. In general, these results are similar to those previously obtained in our laboratory when comparable inhibitors were used to block hyphal damage by neutrophils (2) and by cell-free systems consisting of myeloperoxidase, halide, and hydrogen peroxide (or a peroxide-generating system) or photoactivated rose bengal (21). Similarly, as was the case with neutrophils, two potential nonoxidative fungicidal mechanisms were not apparent in normal monocytes; saturation of iron chelators and addition of polyanions to neutralize cationic proteins did not inhibit damage to hyphae by monocytes. Leukocyte cationic proteins and other granule-associated substances used by other investigators appeared to be responsible for damage to Candida yeasts $(3,30$, 37). In our own studies, partially purified fractions of monocytes rich in lysosomal granules did not damage Candida hyphae. Miller noted that comparable cell homogenates from murine macrophages had no bactericidal activity (38). However, cationic proteins and perhaps other granule constituents as well might act as ancillary antifungal mechanisms, enhancing effects of oxidative mechanisms (39). While these studies have not yet identified all mechanisms active in killing of hyphae by normal monocytes, the data using monocytes from both myeloperoxidase-deficient and one of the four chronic granulomatous disease patients suggest the existence of potent myeloperoxidase-independent or nonoxidative antihyphal systems. Our results indicated that anaerobiasis significantly reduced but did not eliminate damage to hyphae by normal monocytes. This is consistent either with the activity of nonoxidative microbicidal mechanisms (40) or with a partially anaerobic system, a possibility that cannot be eliminated completely. In any case, whether or not nonoxidative antifungal mechanisms are active in normal monocytes, results with myeloperoxidase-deficient cells suggest that myeloperoxidase-independent antifungal mechanisms may be more active in monocytes than in neutrophils. Such mechanisms may be more evident due to compensation in myeloperoxidasedeficient cells compared with normal cells (3). When additional patients with myeloperoxidase deficiency 
become available for study, further insights into alternative oxidative or nonoxidative antihyphal mechanisms should be obtainable using inhibition of myeloperoxidase-deficient monocytes.

Though results of damage to Candida hyphae by neutrophils in prior studies $(1,2)$ and by monocytes in current studies are similar in most respects, there are important distinctions which have been noted above. Defects in leukocyte function have been associated with candidiasis, but monocyte function and neutrophil function do not necessarily correlate (41). In addition, monocytes and monocyte-derived macrophages have important roles in the afferent limb of the immune response, in antigen processing, in secretory functions, and in modulating the immune response (5). Therefore, besides damaging hyphae, the interactions of monocytes with hyphae may have effects on the immune response to Candida that neutrophils do not share.

Electron microscopy also revealed similarities and important differences in interactions of hyphae with monocytes as compared with neutrophils. Both types of phagocytic cell attached to hyphae in the absence of serum. It appeared that monocyte plasma membranes adhered directly to structural portions of hyphal cell walls. Some partially engulfed hyphae were clearly damaged and appeared to be so disrupted that a fungicidal effect must have occurred. However, in contrast to neutrophils, partial engulfment, and damage to hyphae by monocytes appeared to be accompanied by damage to the monocytes themselves. These monocytes appeared swollen and many had disrupted membranes. In addition, many partially ingested and freefloating hyphae had surface-adherent vesicles that were bounded by three-layered unit membranes. Since these vesicular membrane fragments are located outside the hyphal cell wall, they derive most likely from destroyed monocytes, which must have been in contact with this fungal cell previously. These results are consistent with the observations of Cline (42), who noted the lability of human monocytes compared with mature macrophages.

The role of monocyte-mediated damage to Candida hyphae in the intact host remains to be established. However, the ability of leukocytes to damage and probbably kill the tissue-invasive forms of this fungus is likely to be of major importance in defense mechanisms against candidiasis in vivo.

\section{ACKNOWLEDGMENTS}

We thank Elisabeth Huber and A. C. Walstrom for expert technical assistance.

This work was supported in part by grant number AI 15338 from National Institutes of Allergy and Infectious Diseases and Biomedical Research Support grant RR05487 from the Division of Research Grants, National Institute of Health.

\section{REFERENCES}

1. Diamond, R. D., R. Krzesicki, and W. Jao. 1978. Damage to pseudohyphal forms of Candida albicans by neutrophils in the absence of serum in vitro. J. Clin. Invest. 61: 349-359.

2. Diamond, R. D., and R. Krzesicki. 1978. Mechanisms of attachment of neutrophils to Candida albicans pseudohyphae in the absence of serum, and of subsequent damage to pseudohyphae by microbicidal processes of neutrophils in vitro. J. Clin. Invest. 61: 360-369.

3. Lehrer, R. I. 1975. The fungicidal mechanisms of human monocytes. I. Evidence for myeloperoxidase-linked and myeloperoxidase-independent candidacidal mechanisms. J. Clin. Invest. 55: 338-346.

4. Rinehart, J. J., A. L. Sagone, S. P. Balcerzak, G. A. Ackerman, and A. F. LoBuglio. 1975. Effects of corticosteroid therapy on human monocyte function. N. Engl. J. Med. 292: 236-241.

5. Territo, M. C., and M. J. Cline. 1977. Monocyte function in man. J. Immunol. 118: 187-192.

6. Leijh, P. C. J., M. T. Van Den Barselaar, and R. VanFurth. 1977. Kinetics of phagocytosis and intracellular killing of Candida albicans by human granulocytes and monocytes. Infect. Immun. 17: 313-318.

7. Peterson, E. M., and R. A. Calderone. 1977. Growth inhibition of Candida albicans by rabbit alveolar macrophages. Infect. Immun. 15: 910-915.

8. Kirkpatrick, C. H., R. R. Rich, and J. E. Bennett. 1971. Chronic mucocutaneous candidiasis. Model building in cellular immunity. Ann. Intern. Med. 74: 955-978.

9. Baehner, R. L., and R. B. Johnston, Jr. 1972. Monocyte function in children with neutropenia and chronic infections. Blood. 40: 31-41.

10. Steigbigel, R. T., L. H. Lambert, Jr., and J. S. Remington. 1974. Phagocytic and bactericidal properties of normal human monocytes. J. Clin. Invest. 53: 131-142.

11. Weiss, S. J., G. W. King, and A. F. LoBuglio. 1977. Evidence for hydroxyl radical generation by human monocytes. J. Clin. Invest. 60: 370-373.

12. Babior, B. M. 1978. Oxygen-dependent microbial killing by phagocytes. N. Engl. J. Med. 298: 659-668, 721-725.

13. Donowitz, G. R., and G. L. Mandell. 1979. Non-oxidative bactericidal activity of normal and C.G.D. monocytes. Clin. Res. 27: 787A. (Abstr.)

14. Böyum, A. 1968. Isolation of mononuclear cells and granulocytes from human blood. Isolation of mononuclear cells by one centrifugation, and of granulocytes by combining centrifugation and sedimentation at Ig. Scand. J. Clin. Lab. Invest. 97(Suppl.): 77-89.

15. Sanderson, R. J., F. T. Shepperdson, A. E. Vatter, and D. W. Talmage. 1977. Isolation and enumeration of peripheral blood monocytes. J. Immunol. 118: 1409-1414.

16. Koski, I. R., D. G. Poplack, and R. M. Blaese. 1976. A non-specific esterase stain for the identification of monocytes and macrophages. In In Vitro Methods in CellMediated and Tumor Immunity. B. R. Bloom and J. R. David, editors. Academic Press, Inc., New York, 359-362.

17. Greaves, M. F., G. Janossy, and P. Curtis. 1976. Purification of human $\mathrm{T}$ lymphocytes using nylon fiber columns. In In Vitro Methods in Cell-Mediated and Tumor Immunity. B. R. Bloom and J. R. David, editors. Academic Press, Inc., New York, 217-229.

18. Kimball, H. R., G. H. Ford, and S. M. Wolff. 1975. Lysosomal enzymes in normal and Chediak-Higashi blood leukocytes. J. Lab. Clin. Med. 86: 616-630. 
19. Klebanoff, S. J., and R. A. Clark. 1977. Iodination by human polymorphonuclear leukocytes: a re-evaluation. $J$. Lab. Clin. Med. 89: 675-686.

20. Dixon, W. J., and F. J. Massey. 1969. Introduction to Statistical Analysis. McGraw-Hill Inc., New York. 3rd edition. 114-119.

21. Diamond, R. D., R. A. Clark, and C. C. Haudenschild. 1980. Damage to Candida albicans hyphae and pseudohyphae by the myeloperoxidase system and oxidative products of neutrophil metabolism in vitro. J. Clin. Invest. 66: 908-917.

22. Klebanoff, S. J. 1970. Myeloperoxidase: contribution to the microbicidal activity of intact leukocytes. Science (Wash. D. C.). 169: 1095-1097.

23. Ouannes, C., and T. Wilson. 1968. Quenching of singlet oxygen by tertiary aliphatic amines. Effect of DABCO. J. Am. Chem. Soc. 90: 6527-6529.

24. Hodgson, E. K., and I. Fridovich. 1974. The production of superoxide radical during the decomposition of potassium dichromate. Biochemistry. 13: 3811-3814.

25. Singh, H., and J. A. Valdez. 1978. Singlet oxygen: a major reactive species in the furocoumarin photosensitized inactivation of $E$. coli ribosomes. Photochem. Photobiol. 28: 539-545.

26. Repine, J. E., J. W. Eaton, M. W. Anders, J. R. Hoidal, and R. B. Fox. 1979. Generation of hydroxyl radical by enzymes, chemicals, and human phagocytes in vitro. Detection with the anti-inflammatory agent, dimethylsulfoxide. J. Clin. Invest. 64: 1642-1651.

27. Gadebusch, H. H., and A. G. Johnson. 1966. Natural host resistance to infections with Cryptococcus neoformans. IV. The effect of some cationic proteins on the experimental disease. J. Infect. Dis. 116: 551-572.

28. Donowitz, G. R., and G. L. Mandell. 1980. Evidence for non-oxidative killing of bacteria by monocytes. Clin. Res. 28: 367A. (Abstr.)

29. Lehrer, R. I., and M. J. Cline. 1969. Leukocyte myeloperoxidase deficiency and disseminated candidiasis: the role of myeloperoxidase in resistance to Candida infection. J. Clin. Invest. 48: 1478-1488.

30. Lehrer, R. I., K. M. Ladra, and R. B. Hake. 1975. Nonoxidative fungicidal mechanisms of human granulocytes: demonstration of components with candidacidal ac- tivity in human, rabbit, and guinea pig leukocytes. Infect. Immun. 11: 1226-1234.

31. Lehrer, R. I. 1978. Metabolism and microbicidal function, pp. 79-82, in Cline, M. J. (moderator): monocytes and macrophages: function and diseases. Ann. Intern. Med. 88: 78-88.

32. Drath, D. B., and M. L. Karnovsky. 1975. Superoxide production by phagocytic leukocytes. J. Exp. Med. 141: 257-262.

33. Johnston, R. B., Jr., C. A. Godzik, and Z. A. Cohn. 1978. Increased superoxide anion production by immunologically activated and chemically elicited macrophages. $J$. Exp. Med. 48: 115-127.

34. Held, A. M., and J. K. Hurst. 1978. Ambiguity associated with use of singlet oxygen trapping agents in myeloperoxidase-catalyzed oxidations. Biochem. Biophys. Res. Commun. 81: 878-885.

35. Harrison, J. E., B. D. Watson, and J. Schultz. 1978 Myeloperoxidase and singlet oxygen: a reappraisal. FEBS (Fed. Eur. Biochem. Soc.) Lett. 92: 327-332.

36. Slivka, A., A. F. LoBuglio, and S. J. Weiss. 1980. A potential role for hypochlorous acid in granulocyte-mediated tumor cell cytotoxicity. Blood. 55: 347-350.

37. Peterson, E. M., and R. A. Calderone. 1978. Inhibition of specific amino acid uptake in Candida albicans by lososomal extracts from rabbit alveolar macrophages. Infect. Immun. 21: 506-513.

38. Miller, T. E. 1971. Metabolic events in the bactericidal activity of normal mouse macrophages. Infect. Immun. 33: $390-397$.

39. Olson, V. L., R. L. Hansing, and D. O. McClary. 1977. The role of metabolic energy in the lethal action of basic proteins on Candida albicans. Can. J. Microbiol. 23: 166-174.

40. Thalinger, K. K., and G. L. Mandell. 1971. Bactericidal activity of macrophages in an anaerobic environment. $J$. Reticuloendothel. Soc. 9: 393-396.

41. Van Der Meer, J. W. M., P. C. J. Leijh, M. Van Den Barselaar, and R. VanFurth. 1978. Functions of phagocytic cells in chronic mucocutaneous candidiasis. $B r$. Med. J. 1: 147-148.

42. Cline, M. J. 1970. Bactericidal activity of human macrophages: analysis of factors influencing the killing of $\mathrm{Lis}$ teria monocytogenes. Infect. Immun. 2: 156-161. 